\title{
Innovative Clinical Investigations: featured papers in IJCARS
}

\author{
Leonard Berliner ${ }^{1}$
}

Published online: 21 March 2018

(c) CARS 2018

It has been the mission of the International Journal of Computer Assisted Radiology and Surgery (IJCARS) to present significant studies relating to computer applications in the fields of radiology and surgery. The focus of many of the published articles may be predominantly technical in nature, but their underlying clinical significance is of paramount importance. As stated in its Aims and Scope, one of the goals of IJCARS is presented here: "To increase the value of health care for the general populace, one focus of IJCARS is on providing information on new diagnostic and therapeutic processes which are facilitated by CARS. This includes providers' experiences, patient outcomes, economic and ethical considerations, and scientific/medical validation results. It is expected that the resulting awareness by users and providers will accelerate the acceptance of CARS-supported procedures in clinical practice" [1].

The Journal, therefore, endeavors to fulfill its mission to not only present new peer-reviewed articles representing the study and analysis of computer methods and applications in medicine, radiology, and surgery, but also to participate in an effort to enhance patient outcomes and to exert a positive influence in achieving both cost-effective and compassionate patient care. IJCARS accepts this responsibility by identifying exceptional articles that meet certain criteria. As presented in the December 2017 issue of IJCARS, articles may be identified as an Innovative Clinical Investigation by demonstrating, with statistical significance, that the material presented will have an impact on diagnostic/therapeutic workflows or that the methods/tools presented will potentially impact clinical outcome for the patient [2].

It is in the nature of the scope and activities of the Journal that the articles published within its pages address issues of the Exploration phase of the IDEAL initiative, as presented in the December 2017 issue of IJCARS [3]. The IDEAL initiative presents a framework for the stages in innovation in surgery or other interventional procedures defined

Leonard Berliner

leb9025@nyp.org

1 Department of Radiology, New York-Presbyterian Brooklyn Methodist Hospital, Brooklyn, NY, USA as idea, development, exploration, assessment, and longterm study (IDEAL). Three aspects of the EXPLORATION stage, in particular, reflect important concerns that contribute to the designation of an Innovative Clinical Investigation in IJCARS: a focus on adverse effects and potential benefits; the importance of learning curves; and the development of definition and quality parameters. In articles selected as Innovative Clinical Investigations, the editors feel that there are lessons or insights within the substance, focus, findings, and/or conclusions that are within the scope of the IDEAL "Exploration."

In this issue of IJCARS, the identification and publication of Innovative Clinical Investigations continues, with seven articles selected by the Editors and given special prominence. For the contributing authors and the readership of the Journal, this process serves several purposes: a reminder that the ultimate goal in biomedical engineering is improved clinical patient care and outcomes; to alert the readership to a special selection of articles in each issue that may be especially important in guiding the way into the future; and to provide recognition and encouragement to investigators who have been successful in bridging the gap between engineering and clinical medicine.

In this issue, new disciplines, such as mixed reality, 3D printing, MRI radiomics and machine learning as well as more established technologies relating to surgical planning and navigation, are addressed in the selected articles. The common threads of interdisciplinary, innovative, and internationality are again maintained. Studies by groups working in Canada, the Netherlands, Germany, the USA, Japan, and Israel are presented.

(1) Mixed reality ultrasound guidance system: a case study in system development and a cautionary tale

G. Ameri ${ }^{1}$, J.S.H. Baxter ${ }^{1}$, D. Bainbridge ${ }^{2}$, T.M. Peters ${ }^{1}$, E.C.S. Chen ${ }^{1}$

${ }^{1}$ Robarts Research Institute, Western University, London, ON, Canada

${ }^{2}$ London Health Sciences Centre, London, ON, Canada 
In mixed reality ultrasound guidance system: a case study in system development and a cautionary tale by G. Ameri, J.S.H. Baxter, D. Bainbridge, T.M. Peters, and E.C.S. Chen, the authors describe the development and testing an ultrasound guiding system for the performance of central line placement via a jugular vein approach. By analyzing the steps taken in designing their system, the authors emphasize the critical importance of proper planning, development, and testing of their system. The authors draw upon their own experiences and the experiences of others in outlining important and appropriate steps in medical product development. This paper not only serves as a roadmap for developing mixed reality projects, but truly any medical system that is supposed to augment or facilitate the performance of medical/surgical procedures.

(2) Positioning error of custom 3D-printed surgical guides for the radius: influence of fitting location and guide design

G. Caiti $^{1}$,J.G.G. Dobbe ${ }^{1}$, G.J. Strijkers ${ }^{1}$, S.D. Strackee $^{2}$, G.J. Streekstra ${ }^{1}$

${ }^{1}$ Department of Biomedical Engineering and Physics, Academic Medical Center, University of Amsterdam, The Netherlands

${ }^{2}$ Department of Plastic, Reconstructive and Hand Surgery, Academic Medical Center, University of Amsterdam, The Netherlands

The authors point out that despite the growing enthusiasm and potential for 3-D printing of surgical cutting guides for orthopedic surgical planning and navigation, there are relatively few studies that (1) extend beyond individual cases; (2) look specifically at the impact of the accuracy of the surgical guides on patient outcomes; and (3) report quantitative measurements of guide positioning errors.

The authors state that navigation errors can arise if the surgical guide is not correctly positioned at the planned bone location, compromising the surgical outcome. In this study, methods for corrective osteotomy of the radius were investigated, with particular attention to the detection, measurement, and analysis of standard and extended guides in proximal, mid, and distal locations along the radius.

(3) Planning of skull reconstruction based on a statistical shape model combined with geometric morphometrics

M.A. Fuessinger ${ }^{1}$, S. Schwarz ${ }^{1}$, C.-P. Cornelius ${ }^{3}$, M.C. Metzger $^{1}$, E. Ellis III ${ }^{4}$, F. Probst ${ }^{3}$, W. Semper-Hogg ${ }^{1}$, M. Gass $^{1}$, S. Schlager ${ }^{2}$

${ }^{1}$ Department of Oral and Maxillofacial Surgery, AlbertLudwigs University Freiburg, Germany
${ }^{2}$ Department of Physical Anthropology, Albert-Ludwigs University Freiburg, Germany

${ }^{3}$ Department of Oral and Maxillofacial Surgery, LudwigMaximilians-University Munich, Germany

${ }^{4}$ Department of Oral and Maxillofacial Surgery, University of Texas Health Science Center, San Antonio, TX, USA

Manual creation of prostheses for cranial defects by a surgeon is a time-consuming process. The authors provide an alternative method of planning prostheses for cranial defects that provides speed and precision. This technique relies on the creation of statistical shape models (SSMs) based on the CT scans of 131 subjects. The authors find that this technique provides greater accuracy than relying on inverse or mirror-image modeling of the opposite cranial hemisphere. An obvious advantage over mirror-image planning is when there are bilateral cranial defects.

(4) Real-time wireless tumor tracking during breast conserving surgery

N. Janssen ${ }^{1}$, R. Eppenga ${ }^{2}$, M.-J. Vrancken Peeters ${ }^{2}$, F. van Duijnhoven $^{2}$, H. Oldenburg ${ }^{2}$, J. van der Hage ${ }^{2}$, E. Rutgers ${ }^{2}$, J.-J. Sonke ${ }^{1}$, K. Kuhlmann ${ }^{2}$, T. Ruers ${ }^{2,3}$, J. Nijkamp ${ }^{2}$

${ }^{1}$ Department of Radiation Oncology, The Netherlands Cancer Institute, Amsterdam, The Netherlands

${ }^{2}$ Department of Surgical Oncology, The Netherlands Cancer Institute, Amsterdam, The Netherlands

${ }^{3}$ Nanobiophysics Group, MIRA Institute, University of Twente, Enschede, The Netherlands

The authors describe surgical navigation system for breast conserving surgery based on real-time tumor tracking using a localization system. This system utilizes real-time and wireless tumor tracking with EM sensors. By integrating preoperative tumor imaging into the surgical procedure, intraoperative localization and guidance toward the actual tumor borders can be achieved. The results suggest that, as compared with other tumor localization techniques, e.g., wire-guided localization (WGL) and radioactive seed localization (RSL), the method presented may achieve a more reliable resection margin and the ability to define the actual tumor volume or shape during the procedure. Specifically, the authors suggest a potential role of the navigation system for resection of diffuse lesions like ductal carcinoma in situ (DCIS), aiming to reduce the high rates of incomplete tumor resections in this patient group.

(5) The role of exposure time in computerized training of prostate cryosurgery: performance comparison of surgical residents with engineering students

P. Joshi ${ }^{1}$, A. Sehrawat ${ }^{1}$, Y. Rabin ${ }^{1}$ 
${ }^{1}$ Department of Mechanical Engineering, Carnegie Mellon University, Pittsburgh, PA, USA

This study demonstrates the feasibility of computer-based training for prostatic cryotherapy, specifically regarding the optimal cryoprobe layout. The authors focus on educational factors influencing the training quality and effectiveness of computerized training for prostate cryosurgery training system. It was found that for computerized training of prostate cryosurgery, that training exposure time (or the time spent working with the system) was more important than the background experience of the test subjects or a specific planning strategy. As computational techniques become more widespread in the treatment of certain conditions, it is important that it is understood how to achieve optimal training with this technology.

(6) Factors related to disagreement in implant size between preoperative CT-based planning and the actual implants used intraoperatively for total hip arthroplasty

T. Ogawa ${ }^{1}$, M. Takao ${ }^{1}$, T. Sakai ${ }^{2}$, N. Sugano ${ }^{1}$

${ }^{1}$ Department of Orthopaedic Medical Engineering, Osaka University Graduate School of Medicine, Japan

${ }^{2}$ Department of Orthopaedic Surgery, Osaka University Graduate School of Medicine, Japan

In total hip arthroplasty, prediction of the optimal implant size is important to prevent perioperative complications. However, it is not easy to achieve complete agreement between the planned size and the actual size required for appropriate implant fit. The authors report that there have been no previous studies that adequately examine the source of discrepancy between planned cup and stem size. In this article, the authors seek to find specific causes for discrepancies. The authors report agreement in implant size was seen for $94.4 \%$ of cups and $85.5 \%$ of stems, respectively. They found that there were no consistent factors responsible for cup size variation. However, stem alignment in the sagittal and coronal planes showed significant differences between the size-matched stem group and the smaller stem group. They conclude that broach alignment should be checked in the coronal and sagittal planes if the intraoperative broach is smaller than the planned size.

(7) MRI radiomics analysis of molecular alterations in low-grade gliomas

B. Shofty ${ }^{1,2}$, M. Artzi ${ }^{2,3}$, D.B. Bashat ${ }^{2,3,5}$, G. Liberman ${ }^{4}$, O. Haim ${ }^{1}$, A. Kashanian 1,2, F. Bokstein ${ }^{2,6}$, D.T. Blumenthal $^{2,6}$, Z. Ram $^{1,2}$, T. Shahar ${ }^{1,7}$
${ }^{1}$ Division of Neurosurgery, Tel Aviv Sourasky Medical Center, Israel

${ }^{2}$ Sackler Faculty of Medicine, Tel Aviv University, Israel

${ }^{3}$ The Functional Brain Center, Tel Aviv Sourasky Medical Center, Israel

${ }^{4}$ Department of Chemical Physics, Weizmann Institute of Science, Rehovot, Israel

${ }^{5}$ Sagol School of Neuroscience, Tel Aviv University, Israel

${ }^{6}$ Neuro-Oncology Service, Tel-Aviv Medical Center, Israel

The role of tumor biomarkers, such as those relating to immunohistochemistry and genetic mutations, is becoming increasingly important in establishing the diagnosis, prognosis, and treatment options for many malignancies. In general, these determinations require tissue sampling and, in the case of low-grade gliomas, require craniotomy. The authors report on the use of MRI radiomics to detect and identify specific biomarkers for low-grade gliomas, specifically those with IDH1-mutated tumors and a genetic analysis for $1 \mathrm{p} / 19 \mathrm{q}$ deletion.

It was found that radiomic analysis was able to differentiate tumors with $1 \mathrm{p} / 19 \mathrm{q}$ intact (astrocytomas) from those with $1 \mathrm{p} / 19 \mathrm{q}$ codeleted (oligodendrogliomas).

\section{Compliance with ethical standards}

Conflict of interest The author declares that he has no conflict of interest.

\section{References}

1. http://www.cars-int.org/cars_journal/journal_of_cars.html?L= $\% 252 F \% 2 F R K \% 3 D 0$. Accessed 3 Nov 18

2. Lemke HU (2017) Editorial for clinical investigations: toward the vision and spirit of CARS with innovative clinical investigations. Int J CARS 12(5):789-793. https://doi.org/10.1007/s11548-017-15865

3. Lemke HU (2017) Editorial for innovative clinical investigations: example of a framework for determining the stages of innovation in surgery or other interventional procedures. Int J CARS 12:20332037. https://doi.org/10.1007/s11548-017-1685-3 